\title{
ABSTRACTS OF PAPERS
}

\section{SUBMITTED FOR PRESENTATION TO THIS SOCIETY}

The following papers have been submitted to the Secretary and the Associate Secretaries of the Society for presentation at meetings of the Society. They are numbered serially throughout this volume. Cross-references to them in the reports of the meetings will give the number of this volume, the number of this issue, and the serial number of the abstract.

334. Dr. A. B. Brown and Professor B. O. Koopman: Covering of analytical loci by complexes.

The purpose of this paper is to prove that the locus defined by the vanishing of analytic functions of $n$ variables is a complex of analysis situs. While the truth of this theorem has been recognized more or less generally, no rigorous proof has been given in the past. Van der Waerden has given a proof in the algebraic case, which has no direct generalization. Lefschetz has sketched a proof in the general case by methods which, however, would have to be profoundly altered before the proof could be considered rigorous. The treatment given in the present paper applies both in the real and in the complex domain, both in the small and in the large. In the real case the $(n-1)$-dimensional part of the locus is either vacuous or an orientable $(n-1)$-cycle $(\bmod 2)$. (Received October 9, 1931.)

335. Dr. Lulu Hofmann: On the double-point configurations of two projective planes on the same base as the result of the superposition of two distinct projectively related planes of euclidean space.

The double-point configuration of two projective planes on the same base is thought of as the result of superposing two projectively related distinct planes of euclidean space. The different types of projective relations are classified from the metric point of view, and examined as to their capability of producing the various double-point configurations, when the planes are superposed in all possible ways. The most interesting and important configuration is the socalled "parabolic" one, consisting of one point and one line which are incident. It is shown that this configuration can be produced in $2 \infty^{1}$ ways for any nonaffine projectivity, in $2 \infty^{2}$ ways for a special type of non-similar affinity, and in no way for any other projectivity. For a general non-affine projectivity, the points and lines that can occur as the elements of a parabolic configuration are all the points on two parallels to the vanishing line except their infinite point, and all the lines on a central line conic except the two parallel to the vanishing line. (Received October 9. 1931.) 
336. Dr. Wladimir Seidel and Dr. S. B. Littauer (National Research Fellows): On the lines of Julia of entire functions. Part I.

In this paper the authors consider the relations between the lines of Julia of an entire function and (1) the asymptotic values attained by $f(z)$ on rays; (2) the order of $f(z)$ on a ray; (3) the type of $f(z)$ on a ray. Among the results obtained we may mention the following two: (I) If the entire function $f(z)$ attains on the rays $\theta=\theta_{1}$ and $\theta=\theta_{2}$ the same finite asymptotic value $a$, and if the rays $\theta=\theta^{\prime}, \theta_{1}<\theta^{\prime}<\theta_{2}$, and $\theta=\theta^{2}, \theta_{2}<\theta^{2}<\theta_{1}+2 \pi$, are lines of Julia, then the two nonalgebraic singularities of the inverse function corresponding to the values a attained on $\theta=\theta_{1}$ and $\theta=\theta_{2}$ respectively are distinct. If the type $\mu\left(\theta_{0}\right)$ of $f(z)$ of order $\rho$ on the ray $\theta=\theta_{0}$ be defined as $\mu\left(\theta_{0}\right)=\lim \sup _{r \rightarrow \infty}\left(\log \left|f\left(r e^{i \theta_{0}}\right)\right| / r^{\rho}\right)$ on the ray $\theta=\theta_{0}$ we call $f(z)$ of vanishing type of order $\rho$ if there exist rays $\theta=\theta_{n}$ converging toward $\theta=\theta_{0}$ on which $f(z)$ is of order $\rho$ and positive type and such that $\lim _{n \rightarrow \infty} \mu\left(\theta_{n}\right)=0$. We prove (II) Every ray on which the entire function $f(z)$ is of vanishing type and order $\rho$ is a line of Julia. (Received October 7, 1931.)

337. Dr. Wladimir Seidel and Dr. S. B. Littauer (National Research Fellows): On the lines of Julia of entire functions. Part II.

In this paper the authors consider necessary criteria and sufficient criteria for the existence of lines of Julia of entire functions. They relate the cluster values along rays and a type of continuity to the existence of lines of Julia. Of the results the following one may be mentioned: Let $f(z)$ be an entire function of the variable $z=r e^{i \theta}$, and let $\theta=\theta_{0}$ be a ray on which $f(z)$ does not assume values everywhere dense in the plane. Consider some angle $\theta_{0}-\delta \leqq \theta \leqq \theta_{0}+\delta$, $\delta>0$, about $\theta=\theta_{0}$ and the region $R: 1<r<2, \theta_{0}-\delta<\theta<\theta_{0}+\delta$. Consider the family of functions $f_{n}(z)=f\left(\sigma_{n} z\right)$ in $R$, where $\left\{\sigma_{n}\right\}$ is an arbitrary monotonic sequence of positive numbers converging to infinity. Then, the necessary and sufficient condition that $\theta=\theta_{0}$ be a line of Julia of $f(z)$ is that at least one of the families of functions $\left\{f_{n}(z)\right\}$, constructed in the manner described above, be nonnormal in at least one point of the region $R$. (Received October 6, 1931.)

338. Professor J. M. Thomas: The condition for an orthonomic differential system.

This paper develops a method of testing whether a given finite set of partial derivatives can be placed in a specified order by assigning integral cotes to independent and dependent variables in Riquier's manner. The treatment involves the discussion of certain systems of linear inequalities. There results a test for determining whether a given system of partial differential equations is orthonomic. (Received September 22, 1931.)

339. Mr. R. M. Foster: A table of Fourier integrals for practical applications. Preliminary report.

Closed form evaluations of Fourier integrals have been studied, in the exponential form, preparatory to the compilation of a comprehensive table. This 
work has included a search of the literature, direct evaluations to fill in some of the obvious gaps, and a systematic investigation of the domains of the parameters for which the formulas are valid. For the most part, the functions included have been those derivable as special cases of the confluent hypergeometric functions, such as the Bessel functions and the parabolic cylinder functions. The restrictions on the parameters have been stated for the validity of the formulas in terms of Riemann integration; it may prove desirable to extend this to Lebesgue integration. (Received October 10,1931.)

340. Dr. V. V. Latshaw: The algebra of self-adjoint boundary value problems.

The Sturmian boundary conditions associated with a fourth-order differential equation are of two distinct types. There may be three boundary conditions at one point and one at the other, or, as in the case of the vibrating bar, there may be two at each point. H. T. Davis has pointed out that the boundary conditions for the first case can never be self-adjoint, while in the second case only two conditions on the coefficients are necessary for selfadjointness. The purpose of the present study has been to state the corresponding facts for systems of any order. A theorem due to Dunham Jackson was used in calculating explicit conditions for self-adjointness of systems of any order with any admissible number of boundary conditions at each point. Applications to special cases exhibit the power inherent in the formulas derived. (Received October 6, 1931.)

341. Dr. Wladimir Seidel (National Research Fellow): Note on analytic curves everywhere dense in the plane.

The author constructs an example of an analytic curve, $w=\sum_{n=0}^{\infty} a_{n} r^{n}, r$ real, $0 \leqq r<1, a_{n}$ complex, having the property that it passes through an everywhere dense set of points in the w-plane. The example is obtained by making use of elliptic functions and conformal mapping. (Received October 6, 1931.)

342. Dr. Lulu Hofmann: On a certain line locus associated with the iines of two distinct projectively related planes in euclidean space.

In one of two distinct projectively related planes of euclidean space, a line $l$ is fixed and the planes superposed in all possible ways so that $l$ and its homologue in the other plane coincide. Then the locus $Q_{l}$ of all lines is determined, which in some one of these superpositions coincide with their homologues. For a non-affine projectivity and a line $l$ not parallel to the vanishing line, nor incident with a focus of its plane, $Q_{l}$ consists of two parabolas. They are both tangent to $l$, a certain perpendicular to $l$, and the vanishing line, and lie on opposite sides of this line. The angle between their axes is bisected by $l$. In all cases above excluded, $Q_{l}$ consists completely or partially of pencils of lines. The present problem was suggested by the author in her paper On a certain metric aspect of plane projective transformations (this Bulletin, vol. 35, pp. 391-400) as, in a certain sense, the plane dual of the problem there discussed. (Received October 9, 1931.) 


\section{Professor L. E. Ward: A third-order irregular boundary value problem and the associated expansion.}

The differential system considered is $d^{3} u / d x^{3}+\left[\rho^{3}+r(x)\right] u=0, u(0)=u^{\prime}(0)$ $=u(\pi)=0$, where $r(x)$ is given by a convergent power series in $x^{3}$ and is such that, when the radius of convergence of this series is less than $\pi, r(x)$ is continuous in the interval $0 \leqq x \leqq \pi$. An integral equation for $u(x, \rho)$ equivalent to the differential system is used to obtain the asymptotic forms of $u(x, \rho)$ in certain sectors of the $\rho$-plane for $x$ in the interval $(0, \pi)$. The asymptotic expressions for the characteristic numbers and characteristic functions are then obtained. It is proved that any function equal to an infinite series of the characteristic functions with constant coefficients which converges uniformly in any subinterval of $(0, \pi)$ can be expressed in the form $x^{2} \phi\left(x^{3}\right)$, where $\phi\left(x^{3}\right)$ is a convergent power series in $x^{3}$. In the proof the asymptotic forms of $u(x, \rho)$ in certain sectors of the $x$-plane for $\rho$ positive and large are found and used, the expansion theorem is proved by use of the Birkhoff contour integral, and an integral equation similar to that for $u(x, \rho)$ is used to obtain asymptotic expressions for certain parts of the integrand. Conditions are given for $x^{2} \phi\left(x^{3}\right)$ to converge uniformly to $f(x)$. (Received September 18, 1931.)

\section{Mr. C. A. Lovell: Affine geometries which are metric.}

This paper makes use of the theory of the linear dependence of functions to obtain a necessary and sufficient condition for the existence of a tensor of second rank whose covariant derivative, formed with respect to a given connection, is zero, which condition is expressed in terms of the given connection alone. The discussion for the general $n$ is made without restriction as to symmetry. This general result is applied to the case $n=2$ and the symmetric case $n=3$. In addition to this, the paper contains a proof for the positive definite symmetric case $n=3$ of the existence of a system of orthogonal geodesic coordinates. A sufficient condition for a special case is given because it gives rise to the idea of reciprocal surfaces. A condition for $n=2$ that the geometries of a system of paths be projectively equivalent to a Riemannian space is also given. (Received October 8, 1931.)

345. Professor E. D. Meacham: The determination of the canonical pencils by means of the linear complexes osculating the asymptotic curves of a surface.

At a point $P_{x}$ on a surface $S$, consider the two linear complexes $C^{\prime}$ and $C^{\prime \prime}$, osculating the asymptotic curves on $S$, and a point $P_{y}$ in an arbitrary plane (not containing $P_{x}$ ) through the reciprocal polar of the projective normal to $S$ with respect to the quadric of Lie. The null planes of $P_{y}$ with respect to $C^{\prime}$ and $C^{\prime \prime}$ meet the asymptotic tangents to $S$ in four points which taken in pairs with $P_{y}$ determine planes the loci of whose null points in $C^{\prime}$ and $C^{\prime \prime}$, as $P_{\nu}$ varies, are either planes or quadrics. This paper is principally an investigation of these quadrics. The determination and generation of the canonical pencils are by-products of the results obtained. (Received September 18, 1931.) 
346. Professor E. D. Meacham: The class of surfaces whose osculating ruled surfaces belong to linear complexes.

By means of Wilczynski's canonical form and local semicovariant tetrahedron of reference, the author in his thesis (Chicago, 1922) studied certain properties, and gave a method of construction, of non-ruled surfaces whose osculating ruled surfaces are contained in linear complexes. In this paper additional properties of this class of surfaces are obtained by using Fubini's canonical form and local covariant tetrahedron of reference. (Received September 18, 1931.)

347. Professor E. D. Meacham: Certain tetrahedrons associated with a point of a non-ruled surface.

Wilczynski associated with a point on a surface four osculating linear complexes. This paper is concerned with an investigation of the properties of five skew quadrilaterals, lying on the quadric of Lie, determined by the directrices of the linear congruences common to pairs of these complexes. Four of the quadrilaterals have been previously studied individually, but the properties of the other, and those which the five have in common, are believed to be new. (Received September 18, 1931.)

348. Professor Edward Kasner: A curiosity in complex geometry.

The fact that the limit of the ratio of arc to chord for analytic non-real curves is not always unity was discussed by the author in this Bulletin, vol. 20 (1914), p. 524, and the application to relativity theory was given in a note in Nature, December, 1921, p. 434. In the plane a discrete set of values 1.00 , $0.94,0.86, \cdots$ may be assumed, but in space of three or more dimensions all values are possible. It is now shown that for regular surfaces, in general, the limit in question, for the curves drawn at a regular point, may assume any value, real or complex, including 0 and $\infty$ with the single exception of $3^{1 / 2} / 2=0.86$. (Received October 5,1931 .)

349. Professor J. D. Tamarkin: On the compactness of the space $L_{p}$.

In connection with a recent paper by Kolmogoroff (Göttinger Nachrichten, 1931, pp. 60-63) a set of necessary and sufficient conditions is derived for the compactness of a set of functions defined over $n$-dimensional euclidean space and integrable together with the $p$ th powers of their absolute values. (Received October 9, 1931.)

350. Professor O. D. Kellogg and Miss Mildred Sullivan: On the derivatives of potentials on the boundary.

The current theorems on the existence, as limits, and the continuity, on the boundary, of potentials of simple and double distributions on surfaces, impose heavier conditions on surfaces and densities than are necessary. The present paper is devoted to an exploitation of a condition employed by Dini, which 
demands less of these data than the usual Hölder conditions. The results are similar to those given in a paper by Professor Kellogg in the Transactions of this Society, vol. 33 (1931), pp. 486-510, with the difference that there the harmonic functions considered were determined by their boundary values, while in the present case they are determined by the densities of the distributions producing them. (Received October 7, 1931.)

\section{Professor Oystein Ore: Formal theory of linear differen- tial equations.}

The present paper contains a new representation of the formal theory of linear differential polynomials, which is in various ways simpler and more complete than the theories of Blumberg, Loewy, and others. In this paper the main properties of differential polynomials including the decomposition theorem of Landau-Loewy will be considered. It might be observed that the same method applies to all non-commutative domains with one- or double-sided Euclid algorithm. (Received October 7, 1931.)

\section{Professor H. M. Gehman: Concerning sequences of homeomorphisms.}

This paper considers the problem of determining conditions under which a homeomorphism exists between the limiting sets of two sequences of continua, corresponding members of which are homeomorphic. In this connection much use is made of a condition of uniformity of approach. Certain theorems of realvariable function-theory are proved as special cases of the theorems of this paper. The following theorem is typical. Given a sequence $\left(M_{i}\right)$ of closed sets, each member of which contains the following set. Let $M$ denote the set of points common to all the sets of the sequence. If (1) $N$ is a set which is homeomorphic with each set $M_{i}$, the homeomorphism being uniform with respect to $N$, and (2) the set of points corresponding to each point $p$ of $N$ has a sequential limiting point $P$ which is not the sequential limiting point corresponding to any other point of $N$, then there exists a homeomorphism $T$ such that $T(N)=M$. (Received October 10, 1931.)

353. Dr. T. C. Benton: Acyclic, bounded, continuous curves which are homogeneous except for a zero-dimensional set having a non-dense perfect $\mathrm{w}$ th derived set.

Following the methods of previous papers the author gives constructions for the sets of the type indicated in the title of the paper. There are two main types, depending on whether the branch points of the set are or are not limit points of the set of non-homogeneous points. The constructions are too lengthy to be set out in a short abstract. (Received October 10,1931.)

354. Mr. Hassler Whitney (National Research Fellow): $A$ characterization of the closed 2-cell.

It is shown that if $M$ is a locally connected continuum containing the simple closed curve $J$ such that (1) if $a b$ is any arc in $M$ with just its two end points 
on $J$, then $M-a b$ is not connected, and (2) $J$ is irreducibly homologous to 0 in $M$ (see Vietoris, Mathematische Annalen, vol. 97, p. 464), then $M$ is homeomorphic with a circle and its interior ( $J$ going into the circle). In using the word irreducibly, we mean that (2) does not hold in any proper closed subset of $M$. (2) may be replaced by the condition ( $\left.2^{\prime}\right)$ : Given any $\epsilon>0$ and two points $a$ and $b$ on $J$ dividing $J$ into the two arcs $\alpha$ and $\beta$, there exists a set of points $a_{i j}$ in $M, 1 \leqq i \leqq m, 1 \leqq j \leqq n$, such that we have $a_{1 j}=a, a_{m j}=b, a_{i 1} \subset \alpha, a_{i n} \subset \beta$, Dist $\left(a_{i j}, a_{i, j+1}\right)<\epsilon$, Dist $\left(a_{i j}, a_{i+1, j}\right)<\epsilon$, and $M$ is irreducible in this respect. The proof is largely combinatorial, and is in essence fairly simple. (Received October 10, 1931.)

355. Professor J. L. Walsh: On the overconvergence of certain sequences of rational functions of best approximation.

Suppose $f(z)$ is an analytic function of $z$ whose singularities form a set $E$ one of whose derivatives $E^{(k)}$ is empty. Suppose $C$ is a closed point set with no point in common with $E$. Then a sequence of rational functions $r_{n}(z)$ of respective degrees $n$ of best approximation to $f(z)$ on $C$ such that the poles of $r_{n}(z)$ lie in $E$ converges to the function $f(z)$ over the entire plane except on the set $E$. The convergence is uniform on any closed point set containing no point of $E$, and on any such point set the convergence is better than that of any geometric series. (Received October 28, 1931.)

\section{Professor P. A. Smith: Properties of group manifolds.}

The author obtains some results relative to the structure of $n$-parameter continuous groups. (Received October 30, 1931.)

357. Dr. Eberhard Hopf: Proof of the analyticity of the surface of a rotating fluid in equilibrium.

In this paper we apply some general methods developed elsewhere for the discussion of the analyticity of solutions of non-linear integrodifferential equations to the problem of figures of equilibrium of rotating fluids. The following result, suggested by Poincaré, Liapounoff, and Lichtenstein, is proved: The surface of equilibrium of a rotating homogeneous fluid whose particles gravitate according to Newton's law is analytic at all the points where the apparent force does not vanish. (Received October 31, 1931.)

358. Professor B. A. Bernstein: On proposition *4. 78 of Principia Mathematica.

Whitehead and Russell view proposition *4. 78 of their Principia Mathematica as a proposition not having its "analogue for classes." The present author (1) proves this view to be a mistaken one by deriving $* 4.78$ from the logic of classes, and (2) shows that the fact of logic for which $* 4.78$ is intended cannot be derived from the Principia theory of deduction. (Received October 26, 1931.)

359. Professor Louise D. Cummings: Hexagonal systems of seven lines in a plane.

This paper is concerned with the determination of the non-equivalent sets 
of seven lines in a plane. Six lines forming a hexagon are selected as the initial six; the addition of the seventh line shows that the number of non-equivalent arrangements is exactly eight. The integers showing the numbers of polygons of $3,4,5, \cdots, n$ sides which occur in each of these eight systems are tabulated. The method, developed by Professor H. S. White, of the unique characterization of a line by means of the crossings of contiguous line-segments in a system, has been used to determine the "mark" for every line in the eight systems. The seven marks of a system are employed to prove the equivalence or the nonequivalence of two systems and to determine the substitution connecting two equivalent systems. (Received October 15, 1931.)

\section{Professor E. T. Bell: On the real multiplication of elliptic functions.}

An explicit solution of the difference equation which occurs in the real multiplication of elliptic functions, and which is equivalent to the problem of real multiplication, is given. This is the equation to which Cayley devoted several papers without arriving at a solution. The equation is in two variables with variable coefficients. It is found that the variables are separable, and thereby the complete solution is easily attainable. This paper will appear in the Annals of Mathematics. (Received October 16, 1931.)

\section{Professor W. M. Whyburn: On the integration of un- bounded functions.}

In a former paper in this Bulletin (vol. 37, pp. 561-564) the author established a relation between the integrals of $F$. Riesz and Lebesgue. The present paper extends the main theorem of the former paper to obtain a necessary and sufficient condition for the measurability of an unbounded function. A new necessary and sufficient condition for Lebesgue summability is given. A definition of an integral of an unbounded function is given that, although similar to the definitions of Harnack and Denjoy, seems to apply to functions not covered by former definitions. (Received October 23, 1931.)

\section{Dr. D. H. Lehmer: On the distribution of roots of poly-} nomials with integer coefficients.

This note is concerned with polynomials of the form (1) $x^{n}+a_{1} x^{n-1}+\cdots$, where $n>1$, and where the $a$ 's are integers. It is assumed that either (1) has no complex roots, or all its roots lie on the same circle in the complex plane. A new proof is given of Kronecker's elegant theorem that in case this circle is the unit circle, then the roots of (1) are roots of unity. Several consequences of this theorem are given, for example: Every irreducible equation of the type (1) with no complex root must have a root outside the open interval $(-\sqrt{ } 2, \sqrt{ } 2)$. In case all the roots of (1) lie on a circle, its radius is of the form $k^{1 / \delta \epsilon}$ where $k$ is an integer, $\delta$ is the G.C.D. of the subscripts of the non-zero coefficients of (1) and $\epsilon$ is 1 or 2 according as $n$ is odd or even. A necessary and sufficient condition for the arguments of the roots to be commensurable with $2 \pi$ is given. (Received October 29, 1931.) 


\section{Dr. D. H. Lehmer: On certain cyclotomic functions.}

In this paper we study the properties and interrelations of three cyclotomic functions introduced by Schur, Pierce and Poulet. Each is a function of an integer $n$ and a polynomial $f$ with integer coefficients. It is shown that the classes of numbers represented by each of the first two functions are the same. By a combination of the methods employed by Schur and Pierce it is possible to improve the results of each. In case the polynomial $f$ is symmetric, the values of Pierce's function are perfect squares. The roots of these squares turn out to be the values of a function which includes Poulet's function as a special case. This function bears the same relation to Pierce's function that our extension of Lucas' $U_{n}$ does to $x^{n}-1$. Each function is found to form a recurring series so that one may easily calculate the values of the functions for large indices $n$. Conditions on $f$ are found for which corresponding functions increase very slowly. This gives a practical method for the identification of large primes. (Received October 29, 1931.)

364. Mr. J. L. Botsford: Contributions to the theory of multiplepoint integral invariants.

This paper deals with necessary and sufficient conditions for multiple-point integral invariants (A. D. Michal, Transactions of this Society, July, 1927) of the system $d x^{i} / d t=\xi^{i}\left(x^{1}, \cdots, x^{n}\right)$. Previous investigations of projective integral invariants (A. D. Michal, this Bulletin, June, 1931) are extended to multiple-point integral invariants. (Received October $30,1931$.

365. Mr. A. H. Clifford: Continuity and differentials of implicit functionals.

Sufficient conditions have been given (Volterra, Fonctions de Lignes; proved in detail by G. C. Evans, Colloquiun Series, Vol. V., Part I.) that a functional equation of the type $H^{x}\left[\phi^{t}, f^{t}\right]=0$ (where $H$ is a functional of the two continuous functions $\phi^{x}, f^{x}$ and depends continuously on the real variable $x$ ) defines $\phi^{v}$ implicitly as a functional of $f$. In this paper the continuity of the implicit functional thus defined is established, and, with additional hypotheses regarding the differentials of $H$, the implicit functional is shown to admit Frechet differentials of a special type (Schmidt integral power forms) out to any order $r$, or of all orders. These results are immediately applicable to the inverse of a functional transformation, i.e., to the solution $\phi^{x}\left[f^{t}\right]$ of an equation of the type $f^{x}=$ $F^{x}\left[\phi^{t}\right]$. (Received October 30, 1931.)

366. Professor Raymond Garver: Concerning certain root location theorems.

This paper is concerned with relations existing between the roots of an algebraic equation and those of its derived equation. First, a number of known results, including the Gauss-Lucas theorem, Jensen's theorem, and certain results of Walsh, Nagy and Mitchell are proved with the aid of simple transformations. The possibility of employing transformations in theorems of this type has been mentioned a few times in the literature, but the method has not been used extensively. Second, a new type of problem is contemplated, namely the 
determination of the maximum value of the ratio of the area of the smallest convex polygon enclosing the roots of the derived equation to the area of the smallest convex polygon enclosing the roots of the given equation. For quartic equations with real coefficients this ratio is always less than $3 / 4 \sqrt{ } 2$, but can be made arbitrarily close to this value. (Received October 30,1931.)

367. Mr. R. S. Martin: The explicit determination of the most general linear continuous functional transformation preservative of continuity.

The Stieltjes kernel of the most general linear continuous functional transformation which transforms continuous functions of a single variable into continuous functions of a single variable is completely characterized. Extensions to the transformations of functions of many variables are also considered. Explicit expression for the transformation of the kernel of a continuous linear form under functional transformations preservative of continuity is given together with sufficient conditions that expression be possible in terms of Riemann-Stieltjes integrals. (Received October 30, 1931.)

368. Mr. R. S. Martin: A theorem of the limits of Stieltjes integrals.

The writer proves by elementary methods that if a sequence of functions $y_{n}(x)(a \leqq x \leqq b)$ each integrable Riemann-Stieltjes with respect to a function $\alpha(x)$ of limited variation on $(a, b)$, converges in any manner to a function $y(x)$ also integrable with respect to $\alpha(x)$, then $\lim _{n-\infty} \int_{a}^{b} y_{n}(x) d \alpha(x)=\int_{a}^{b} y(x) d \alpha(x)$. (Received October 30, 1931.)

369. Mr. R. S. Martin: Representation of function valued polynomial functionals of a continuous function.

The writer considers the representation by multiple Stieltjes integrals of function valued polynomial functionals of a continuous function of a single variable. The Stieltjes kernel corresponding to the most general polynomial which for continuous argument is a continuous function is completely characterized. (Received October 30, 1931.)

370. Mr. R. S. Martin: Function valued analytic functionals of several functions.

Function valued analytic functionals of several continuous functions are defined by means of their expansion in a series of multiple Stieltjes integrals. A notation whose manipulation is analogous to that of umbral indices is introduced and the processes justified. Application of the results is made in the solution of completely integrable systems of total functional-differential equations. (Received October 30, 1931.)

371. Mr. R. S. Martin: Contribution to the theory of analytic functions in abstract spaces. Preliminary report.

The writer employs a modification of Banach's postulational definition of an abstract vector space, replacing the condition of closure under multiplica- 
tion by real numbers by the condition of closure under multiplication by numbers of a given ring. Some properties of analytic correspondences between the elements of such spaces are studied relative to the choice of the ring. In particular, if the space is closed under multiplication by all complex numbers, analytic correspondences have properties analogous to those of ordinary analytic functions which they include as a special case. (Received October 30,1931.)

372. Mr. Harry Matison and Mr. A. H. Levine: Integral addition theorems for Bessel functions.

The Cauchy problem of the equation $\partial^{2} u / \partial t^{2}-\partial^{2} u / \partial x^{2}-u=0$ gives rise to a pair of functional transformations on two functions. These transformations involve Bessel function of orders zero and one. The fact that these transformations form a continuous group when $t$ is regarded as a parameter leads to identities involving repeated intergrals of Bessel functions and two arbitrary functions. After a series of transformations, these are found to imply a set of twelve identities involving only Bessel functions and integrals of Bessel functions. Finally this set of twelve identities is shown to be equivalent to the fact that the transformations considered form a group. (Received October 30,1931.)

373. Professor A. D. Michal: Parallel displacement of a multiple-point tensor along several curves in Riemannian geometry.

The notion of a multiple-point tensor was introduced by the author in connection with his investigations on functional invariants (Transactions of this Society, 1927, pp. 612-646). The present paper deals with a certain parallel displacement of a multiple-point tensor along several curves in Riemannian geometry. This definition reduces to that of Levi-Civita for the case of the onepoint tensor (usual type of tensor). (Received October 31,1931.)

374. Professor O. W. Albert: Relations between the projective and metric differential geometries of surfaces.

In this paper we compare the Gauss equations of metric geometry for a special oblique system of axes with the differential equations of projective geometry of a surface used by Professor Wilczynski (Transactions of this Society, vol. 8) and determine relations between the homogeneous coordinates of a point and its Cartesian coordinates referred to our special trihedral. We get simpler metric equations of the osculating linear complexes of the asymptotic curves, $C^{\prime}$ and $C^{\prime \prime}$, given first by Morrison (American Journal of Mathematics, vol. 39). We then find similar equations for the osculating linear complexes of the osculating ruled surfaces, $C_{1}$ and $C_{2}$, and determine the directrices of their congruence and the conditions for these directrices to be real, to be parallel to or perpendicular to the surface normal. We show that the directrices of the congruence of $C^{\prime}$ and $C^{\prime \prime}$, the directrices of the special congruence of $C_{1}$ and $C_{2}$, and the tangents to the asymptotic curves are the six edges of a tetrahedron.

By studying the other congruences determined by the four complexes, we find the metric conditions on the invariants of $C_{1}$ and $C_{2}$ which make the directrices real, imaginary or coincident. We also find the metric conditions for the directrices of the congruences of the four complexes to determine a linear 
congruence whose directrices are the two lines common to the four complexes. (Received October 31, 1931.)

\section{Professor A. A. Shaw: An overlooked numeral system of antiquity.}

In this paper the author gives an account of the Armenian alphabetic numerals used in the fourth and subsequent centuries of our era. The Armenians used the first nine letters of their alphabet to represent units, the second nine to represent tens, the third nine to represent hundreds, and the fourth nine to represent thousands. This arrangement agrees with the Semitic and the Greek alphabetic systems of numeration. But the works of Ananiah Shiragooni (first quarter of the seventh century, A.D.) reveal variant forms of numbers involving multiplicative and additive principles which reduce the thirty-six symbols to twelve and place the Armenian notation on a decimal basis without use of zero. The corresponding variant forms do not exist in the Semitic and the Greek systems. The paper closes with a few illustrations involving the four fundamental operations. (Received October 30,1931.)

\section{Professor A. R. Williams: A transformation of the lines of space effected by means of two quadratic reguli.}

If we take two quadratic reguli which do not lie on the same quadric, and two generators of each regulus of general position, the Plücker coordinates, $p_{i k}$, of either of the two transversals of these four generators are cubic functions of the coordinates of the other. The special lines which do not have unique transforms constitute a congruence. The use of a quartic scroll in the same manner suggests itself, but leads only to a linear transformation on the line coordinates, since the generators of the scroll belong to a certain linear complex. (Received November 2, 1931.)

\section{Professor B. C. Wong: Enumerative properties of r-space} curves.

In the study of enumerative properties of algebraic curves in space of $r$ dimensions it is often convenient to decompose a curve into two or more component curves and to study these curves both individually and in relation to one another. In this paper the writer makes a systematic study of the skew $m$ sided polygon with $m-1+p$ vertices, or the connected $m$-line with $m-1+p$ incidences into which a curve $C_{p}{ }^{m}$ of order $m$ and deficiency $p$ has been completely decomposed, and obtains numerous new results for $C_{p}{ }^{m}$, some of which are difficult to obtain otherwise. (Received November 2, 1931.)

\section{Professor Lincoln La Paz: Variation problems of which the extremals are minimal surfaces.}

In this paper it is shown that the partial differential equation $F(x, y, z, p, q, r$, $s, t) \equiv\left(1+q^{2}\right) r-2 p q s+\left(1+p^{2}\right) t=0$ admits a multiplier $M(x, y, z, p, q) \neq 0$, unique up to a constant factor, for which the equivalent equation $M F=0$ has a selfadjoint equation of variation. This result, in sharp contrast to that obtained by Darboux in his study of the analogous problem for the ordinary differential 
equation $y^{\prime \prime}-\phi\left(x, y, y^{\prime}\right)=0$, is shown to be due to the special form of the function $F$. The most general problem of minimizing $I=\iint f(x, y, z, p, q) d x d y$ associated with the equation $M F=0$ is found to have the integrand function $h=\left(1+p^{2}+q^{2}\right)^{1 / 2}+\partial w_{1} / \partial x+\partial w_{2} / \partial y$ where $w_{1}$ and $w_{2}$ are arbitrary differentiable functions of $x, y$ and $z$ alone. (Received November 4, 1931.)

\section{Dr. Ben Dushnik: On the Stieltjes integral.}

This paper presents the results of studying the application of a general limiting process (as first defined explicitly by E. H. Moore and H. L. Smith, $A$ general theory of limits, American Journal of Mathematics, vol. 44, 1922, pp. 102-121) to an additive many-valued number-valued set function. An example of such a set function would be the sums considered in the classical definition of the Riemann and Stieltjes integrals; additional examples are found in this paper. The limit of such a set function when it exists (as a finite number) is called the integral of the set function. The theorems developed include one on integration by parts, thus solving a problem of Pollard (The Stieltjes integral and its generalizations, Quarterly Journal of Mathematics, vol. 49, 1920-23), and several which concern themselves with sequences of set functions. When the additional examples of set functions are considered, there are demonstrated a few existence theorems, as well as certain other theorems whose main trend is to generalize some of the known properties of the Stieltjes integral. (Received November 3, 1931.)

380. Professor C. N. Moore: On the absolute convergence of the developments in Bessel's functions.

The convergence and uniform convergence, summability and uniform summability of the developments in Bessel's functions have been studied by various writers. The case of absolute convergence, however, seems to have received little or no attention. In the present paper certain asymptotic formulas for the coefficients of these developments, obtained by the writer in a series of previous papers, are used to derive various sets of sufficient conditions for absolute convergence. As would naturally be expected, it is found that somewhat more stringent requirements are needed than in the case of Fourier series. (Received November 5, 1931.)

381. Professor W. D. Baten: Frequency laws for the sum of $n$ variables which are subject each to given frequency laws.

This article develops frequency laws for the sum of $n$ independent variables which have known frequency laws. These functions for the sum are derived by Dodd's method. Relations between measures of precision for the sum function and the measures of precision for the individual frequency functions are treated. A certain definite integral, which arises in developing frequency laws for the sum, is evaluated by integrating around a certain contour in the complex plane. Certain frequency functions are expanded into finite series similar to Charlier's series. A type 3 curve is obtained for the law for the sum of the squares. (Received November 3,1931 .) 


\section{Professor H. T. Davis: Polynomial approximation by the method of least squares.}

In this paper the author considers the problem of fitting polynomials from the first to the seventh degree inclusive to data given over a discrete range from $x=-p$ to $x=+p$. The method of finding polynomials which are orthogonal for discrete summation instead of continuous integration is employed and an explicit set of polynomials is calculated. These are shown to agree essentially with polynomials previously found by J. P. Gram (Journal für Mathematik, vol. 94, 1883, pp. 41-73). Some properties of these polynomials are exhibited and elaborate tables are computed which facilitate the practical application of the methods. This paper is an extension of one on a similar subject published by Dr. V. V. Latshaw and the author in the Annals of Mathematics, vol. 31 (1930), pp. 52-78. (Received November 4, 1931.)

383. Professor Saul Pollock: On the determination of formulae for the orders of line loci in five-space by graphic representation.

Graphic representation (introduced by B. C. Wong, On the number of apparent triple points of surfaces in space of four dimensions, this Bulletin, vol. 35, 1929 , pp. 339-343), is a method by decomposition based on the principle of preservation of number. By means of specialized Cartesian systems for fourspace and graphic systems for five-space, the Wong method may be extended to the determination of the orders of the ruled loci in five-space whose elements are incident with given curves, surfaces, and hypersurfaces, and satisfy certain conditions. The purpose of this paper is to show how these formulae may be derived by an extension of the Wong theory. (Received November 6, 1931.)

\section{Professor Tibor Radó: On convex regions.}

If the interior of a circle is mapped in a one-to-one and conformal way upon a convex region, then every circle interior to this circle is also carried into a convex curve, according to a theorem of Study. This suggests an investigation of topological transformations carrying circles into convex curves. Every transformation which is sufficiently regular from the point of view of differentiability has this property in the small, as may be easily shown. If a transformation has this property in the large, then it has on the boundary continuity properties similar to those of conformal transformations. (Received November 4, 1931.)

385. Professor R. L. Wilder: On the linking of Jordan continua in $E_{n}$ by $(n-2)$-cycles.

The following is a classical theorem in the theory of Jordan continua (Cf. R. L. Moore, this Bulletin, vol. 29, pp. 295-296; C. Kuratowski, Fundamenta Mathematicae, vol. 6, pp. 140-141): If, in the plane, two points $P$ and $Q$ are separated by a Jordan continuum $M$, then $P$ and $Q$ are separated by a simple closed curve of $M$. In the present note it is shown that if in $E_{n}$ a Jordan continuum $M$ is linked by a cycle $\Gamma^{n-2}$, then $\Gamma^{n-2}$ links a simple closed curve of $M$. For the case $n=2$, the former theorem follows as a special case. (Received November 4, 1931.) 
386. Professor G. Y. Rainich: Covariant form of a special case of Dirac's equations.

It is known that Dirac's equations of the theory of the electron, although they do not appear in covariant form, are invariant under the group of Lorentz transformations. The independent variables in these equations - the $\psi$ 's-are not components of a tensor, but certain quadratic expressions in the $\psi$ 's are. The general problem of which a special case is treated here is to write a system of equations in second degree quantities equivalent to the Dirac equations and to put them in covariant form. The problem is complicated by the fact that the $\psi$ 's are not uniquely determined by the second degree quantities. The special case considered is characterized by stating that the $\psi$ 's are equal in pairs, or, that the two invariants and the six-vector vanish, and the two four-vectors coincide. The second degree quantities reduce to the components of one vector $j$ of zero square. Complicated differential relations for its components are found by painful computation; and then, using the invariance of the system, comparatively simple differential equations are derived in covariant form. They are non-linear in the components of the vector $j$, so that Dirac's equations may be considered as results of linearization of our system. (Received November 4,1931.)

387. Professor Cornelius Lanczos: The electromagnetic field as a natural property of Riemannian geometry.

Einstein's field-equations $R_{i k}=0$ are not to be considered as the simplest characterization of a Riemannian geometry if we require that this geometry should have no singularities. This restriction leads to euclidean geometry only. To generalize these equations we must expect to find field-equations for $R_{i k}$. Supposing that these equations obey a variation principle, we can prove generally that they can be integrated by a free vectorial function $\phi_{i \delta}$. This vector $\phi_{i \delta}$ is determined by the divergence-condition for $R_{i k}$ which must be fulfilled in addition to the field-equations. Selecting the Lagrangian function by the condition that the varied field-integral should not depend on the arbitrariness of the unit of length, we can show that the vectorial function $\phi_{i \delta}$ has the same properties as the vector potential of the electromagnetic field, and we can deduce also the motion-law of Lorentz for an electric particle.. (Received November 3,1931 .)

388. Miss Grace Shover: Class number in a linear associative algebra.

Consider a semi-simple algebra with a principal unit taken over the rational field. If $\beta$ is any number in the left ideal $\Omega$, there exists an integral matrix $M$ such that $S(\beta)=M G$ where $S(\beta)$ is the second matrix of $\beta$ and $G$ is a matrix representation of $\Omega$. The adjoint $M^{A}$ of $M$ belongs to the same minor class as $G$. Using a theorem of Minkowski, we can find a number $\beta$ of $\Re$ such that the determinant of the corresponding $M^{A}$ is bounded by a number which depends only on the algebra. Hence the class number is finite. If $G$ is a left (right) ideal matrix with class matrices $D_{p}$, then $G^{A T} T^{A}\left(G^{A T} T^{A T}\right)$ is a right (left) ideal matrix with class matrices $D_{p}^{T}$ where $G^{A T}$ is the transpose of the adjoint of $G$ and $T$ is the discriminant matrix of the algebra. Hence the right and left class numbers are equal. (Received October 5, 1931.) 
389. Dr. L. E. Bush: Note on the discriminant matrix of an algebra.

C. C. MacDuffee (Transactions of this Society, vol. 33, p. 427) defines a normal basis for a linear associative algebra with a principal unit. In this paper an extension of this definition to a general linear associative algebra is made, and its existence for such an algebra is proved. MacDuffee's theorem 2, which gives a simple relation between the multiplication constants of an algebra with a normal basis, is shown to hold for a general linear associative algebra. (Received October 20,1931.)

390. Dr. L. E. Bush: On the equivalence of the decompositions of an algebra with respect to a principal idempotent.

A definition is given for the equivalence of the decompositions of a linear associative algebra with respect to two different principal idempotents, and from this definition a necessary and sufficient condition for two such decompositions to be equivalent is deduced. Formulae are found which give all the principal idempotents of certain classes of algebras, and it is shown that for an algebra belonging to one of these classes the decompositions with respect to any two principal idempotents are equivalent. A semi-modulus of an algebra is defined and it is shown that the above mentioned property is possessed by every algebra with a semi-modulus. (Received October 20, 1931.)

391. Professor W. E. Roth: On algebraic equations having only real roots.

The present paper proves the following theorem for equations $f(x)=a_{0} x^{n}$ $+a_{1} x^{n-1}+\cdots+a_{n}=0, a_{0} a_{n} \neq 0$, whose roots $x_{i}, i=1,2, \cdots, n$, are all real. If $s_{k}=x_{1}^{k}+x_{2}^{k}+\cdots+x_{n}^{k}$, where $k$ is any integer, and if $a_{i j}{ }^{\lambda}=s_{\lambda(i+j-2)}$, $2 \leqq i+j \leqq 2 h \leqq 2 n$, where $h$ is a positive integer and $\lambda$ any integer not zero, then the determinants $\left|a_{i j}{ }^{\lambda}-x^{\lambda(i+j-2)}\right|$ are negative only on intervals which do not contain roots of $f(x)=0$. Some extensions of this theorem to the location of simple and multiple roots of $f(x)=0$ are developed. (Received October 16, 1931.)

392. Professor R. V. Churchill: Canonical forms for symmetric linear vector functions in pseudo-euclidean space.

Any symmetric matrix has a simple canonical form under orthogonal transformations. Geometrically this form is a canonical form for the symmetric linear vector function or the symmetric second rank tensor in euclidean space. It seems that canonical forms have not been found for matrices under pseudoorthogonal or Lorentz transformations. This is done in this paper for certain matrices with four rows. Geometrical terminology and methods are used. Symmetric linear vector functions in the space of special relativity are classified here into four types according to the values of their invariants, and a canonical form is found for each type. The method used to find these forms is essentially that of finding sets of orthogonal directions whose vectors are transformed in a simple way by the function, and such that when referred to these directions the function can be described by using a minimum number of its independent 
components. Certain properties of the function are made evident by these forms. (Received November 4, 1931.)

393. Mrs. Pearl C. Miller: Four points of intersection of lines drawn from the vertices of a triangle to the points of contact of the nine point circle with the in-and ex-circles.

This paper by means of trilinear coordinates finds the equations of the lines mentioned in the title and the coordinates of their points of intersection, also the equations of the pencil of lines from a single vertex to the four points of the harmonic range, and thus the following theorems are established. Theorem 1: If lines are drawn from the vertices of a triangle to the points of contact of the opposite ex-circles with the nine point circle, and also a line is drawn from the center of the nine point circle to its point of contact with the in-circle, these four lines meet in a point which is the fourth harmonic to the nine point center, the in-center and the point of tangency of the nine point circle with the incircle. Theorem 2: A point having similar properties lies on each of the lines drawn from the nine point center to the points of tangency of the three excircles with the nine point circle. (Received October 26, 1931.)

394. Professor Arnold Emch: The symmetric $(n, n)$-correspondence and some geometric applications.

This paper shows how by means of the completely symmetric or involutorial $(n, n)$-correspondence a large number of geometric problems involving the property of closure may be solved in a manner which is very simple when compared with the arduous labors which are often necessary to accomplish the same result by synthetic means. Among the curves and surfaces studied by this method are the Lüroth quartic, admitting of an infinite number of inscribed pentalaterals, and the Schur sextic in space which admits of $\infty^{1}$ inscribed pentahedrons whose faces envelope a class-cubic and whose edges generate a ruled octavic, its connection with the Lüroth quartic being considered. It is also proved that the Schur sextic is the Jacobian curve of a net of quadrics and that its manifold is $\infty^{20}$ as compared with $\infty^{24}$ for the general sextic of genus 3 (or 4). (Received October 28, 1931.)

\section{Professor J. M. Thomas: Riquier's existence theorems.}

This paper is an addendum to one which appeared previously under the same title (Annals of Mathematics, (2), vol. 30, 1929, pp. 285-310). It indicates how to form the integrability conditions of an orthonomic system one or more of whose left members are derivatives of other left members, a detail omitted from the previous exposition. In addition, the process of forming the complete set of monomials is modified so that the complete set always contains the original set, a property possessed by the complete set as defined by Janet. The process indicated in the present paper, however, still has the advantage of forming the complete and complementary sets directly from the Maclaurin expansion. Finally, Riquier's method of reducing an orthonomic system to passive form is applied to deduce the general theorem on compatibility of systems of total differential equations given by $\mathrm{O}$. Veblen and the author (Annals of Mathematics, (2), vol. 27, 1926, p. 290). 
396. Professor M. A. Basoco: Fourier developments for certain pseudo-periodic functions in two variables.

The functions $\phi_{\alpha \beta \gamma}(x, y) \equiv \theta_{1}^{\prime}\left(\theta_{\alpha}(x+y) / \theta_{\beta}(x) \theta_{\gamma}(y)\right)$, where the $\theta^{\prime}$ 's are Jacobi theta functions and $(\alpha, \beta, \gamma)$ are certain sixteen triads out of the possible ones which can be selected from the numbers $0,1,2,3$, have been studied by Jacobi, Kronecker, Hermite and others. More recently, Bell (Transactions of this Society, 1921; Colloquium series, VII, p. 88) has emphasized their importance in connection with certain arithmetical results. In the present paper we are concerned with certain functions $\Theta_{\alpha \beta \gamma}(x, y)$ related to the above functions by the identity $\Theta_{\alpha \beta \gamma}(x, y)=\theta_{1}^{\prime} / \theta_{\beta}(x) \cdot \phi_{\alpha \beta \gamma}(x, y)$. The arithmetized Fourier developments of these functions are obtained in a manner suitable for use in certain problems in number theory. These developments lead to the consideration of incomplete numerical functions in two variables. So far as the author is aware no expansions involving this type of functions have hitherto appeared in the literature. However, Uspensky (this Bulletin, October, 1930) by purely arithmetic methods has proved certain formulae of the paraphrase type which have reference to incomplete functions in more than one variable. (Received October 21, 1931.)

397. Dr. I. J. Schoenberg: On finite and infinite completely monotonic sequences.

F. Hausdorff (Mathematische Zeitschrift, vol. 16, pp. 220-248) has proved the following theorem: The infinite sequence of numbers $\mu_{0}, \mu_{1}, \mu_{2}, \cdots$, is completely monotonic, i.e., the whole set of inequalities

$$
\Delta^{k} \mu_{l} \equiv \mu_{l}-\left(\begin{array}{l}
k \\
1
\end{array}\right) \mu_{l+1}+\cdots+(-1)^{k} \mu_{l+k} \geqq 0, \quad(k, l=0,1,2 \cdots),
$$

holds, if and only if there is a non-decreasing function $\chi(t)$ for $0 \leqq t \leqq 1$, such that (2) $\mu_{n}=\int_{0}^{1} t^{n} d \chi(t),(n=0,1,2, \cdots)$, holds. The author gives a new proof for this theorem. In order to find the parametric representation (2) for infinite sequences, the analogous problem for a finite sequence $\mu_{0}, \mu_{1}, \cdots, \mu_{p}$, is first solved. Such a sequence is also called completely monotonic if $\Delta^{k} \mu_{l} \geqq 0$ for $k+l \leqq p$. A parametric representation is easily found for the most general solution of this finite set of linear inequalities which, on making $p$ become infinite, yields the parametric representation (2) for the original transcendental problem. The same method permits us to assign a rather large class of infinite sets of linear inequalities of the same type as (1), whose solutions may be represented parametrically by means of Stieltjes integrals. This generalization will be considered in another paper. (Received November 5, 1931.)

398. Professor E. W. Chittenden and Mr. Selby Robinson: Properties of coverings of abstract type related to reducibility.

Chittenden (Transactions of this Society, vol. 31, pp. 290-321) has developed a theory of the reducibility of coverings of abstract type. The present paper extends and perfects this theory with respect to the reducibility of a single covering. The notation and terminology are improved, and new results are found for the case of coverings of irregular power and of regular powers which 
are limits of lower powers. New sets of equivalent properties of coverings are found. These results are applied by Robinson in the study of classes of coverings. (Received November 5, 1931.)

399. Mr. R. M. Foster: The linear graphs associated with electrical circuits.

If all the electrical properties are abstracted from a given electrical circuit, there remains a geometrical circuit, or linear graph, completely characterized by the sets of branches terminating at the various vertices. To meet the conditions of electrical problems the concept of equivalent graphs is extended to include cases where two graphs become homeomorphic upon the interchange of any branches or groups of branches which have a series connection. Enumerations of distinct graphs are given, classified according to nullity (number of branches minus number of vertices plus number of separate parts) and according to rank (number of vertices minus number of separate parts). A study is made of symmetrical graphs, defined as non-separable, regular graphs in which all of the branches, as well as all of the vertices, enter symmetrically. The graphs formed by the edges of the regular polyhedra are symmetrical; many other cases are shown to exist. (Received November 4,1931.)

400. Professor V. W. Adkisson: On extending a continuous (1-1) correspondence of continuous curves on a sphere.

A necessary and sufficient condition that a continuous $(1-1)$ correspondence of two plane continuous curves be extendible to a correspondence of their planes has been given by H. M. Gehman, (Transactions of this Society, April, 1926, pp. 252-265). By a slight modification of the definition of "sides preserved" Gehman's result is extended to continuous curves on a sphere. The following theorem is also established. If $M$ is a cyclicly connected continuous curve lying on a sphere $S$, and $T$ a continuous $(1-1)$ correspondence such that $T(M)=M$, then a necessary and sufficient condition that $T$ be extendible to $S$ is that sense be preserved (or altered) on the simple closed curves of $M$. Sense is defined for simple closed curves on the sphere to conform with the definition for sense in the plane (J. R. Kline, Annals of Mathematics, vol. 19, pp. 185200). (Received November 2, 1931.)

401. Professor L. W. Cohen: On the minors of absolutely convergent determinants.

Let $\Delta=\left|\delta_{i k}+a_{i k}\right|$ be an infinite determinant such that

$$
\begin{gathered}
\sum_{i-1}^{\infty}\left|a_{i i}\right|<+\infty ;\left[\sum_{i=1}^{\infty}\left[\sum_{k=1}^{\infty}\left|a_{i k}\right|^{q}\right]^{p / q}\right]^{1 / p}=\sigma<+\infty(i \neq k) ; \\
1 / p \pm 1 / q=1 ; 1<p \leqq 2 .
\end{gathered}
$$

Let $A_{r_{1}} \ldots r_{\theta} ; c_{1} \ldots c_{s}$ be the minor of $\Delta$ obtained by striking out the rows $r_{i}$ and the columns $c_{i}\left(r_{i} \neq c_{i} ; i=1,2, \cdots, s\right)$. Let $\left[\sum_{k=1}^{\infty}\left[\sum_{i=1}^{\infty}\left|a_{i k}\right|^{p}\right]^{q / p}\right]^{1 / q}=\tau$. Cer tain bounds for the minors are obtained, typical of which is

$$
\left[\sum_{c_{1} \ldots c_{s=1}}^{\infty}\left[\sum_{r_{1} \ldots r_{s}=1}\left|A_{r_{1}} \ldots r_{s} ; c_{1} \ldots c_{s}\right|^{q}\right]^{p / q}\right]^{1 / p} \leqq s ! \sigma^{s} P(1+\tau /(1-\sigma))^{s}
$$

where $r_{i} \neq c_{i}, P=\pi(1+|p|), p$ being a circular product $a_{i_{1} i_{2}} a_{i_{2} i_{3}} \cdots a_{i_{i} i_{1}}$, and the product is extended over all such $p$ 's. (Received November 4,1931 .) 
402. Professor H. H. Conwell: Linear associative algebras of infinite order whose elements satisfy finite algebraic equations.

In this paper an investigation is made of linear associative algebras of infinite order, whose elements satisfy finite algebraic equations with coefficients in the field $\Xi$ over which the algebra is taken. Assuming the existence in the algebra of a set of elements $U$ of such a nature that for every $a \neq 0$ there is determined uniquely a positive integer $n$ and a set of distinct elements $u_{1}, u_{2}, u_{3}, \cdots, u_{n}$ of $U$ and $n$ non-zero elements $\xi_{1}, \xi_{2}, \cdots, \xi_{n}$ of $\Xi$ such that $a=\sum_{i=1}^{2} \xi_{i} u_{i}$, it is shown that many of the theorems of finite linear associative algebras can be extended to non-finite algebras. (Received October 14, 1931).

403. Mr. Michael Goldberg: The isoperimetric problem for polyhedra.

The problem of the determination of the convex polyhedra of greatest volume for a given number $n$ of faces and a given surface area has been treated by Steiner, Lindelöf, Minkowski, Steinitz (Crelle's Journal, vol. 158, 1927, and vol. 159, 1928), but solutions have been obtained only for $n<8$. Lindelöf proved that a necessary condition is that the faces of the polyhedra be tangent to a sphere at the center of gravity of the faces. Let $K=F^{3} / V^{2}$ where $F=$ surface, $V=$ volume. This paper establishes upper and lower bounds for $K$, limiting $K$ to a narrow band of values. Series of polyhedra for which the values of $K$ lie between these bounds are given. An example of a lower bound for $K$ is $K \geqq 6(n-2) \tan A\left(4 \sin ^{2} A-1\right)$, where $A=\pi n /(6(n-2))$. The equality holds for the regular tetrahedron, cube and dodecahedron, showing that they are "best." The regular octahedron and icosahedron are not the "best," and indeed, the paper exhibits "better" polyhedra. (Received September 8, 1931.)

404. Professors Virgil Snyder and Hazel Schoonmaker: Two involutorial transformations, of orders 11 and 9, associated with null reciprocities.

The first transformation discussed by the authors is defined by means of 4 skew lines $a_{i}$, a fixed point $A_{0}$ not on any $a_{i}$, and 5 fixed lines of a pencil $g$. A plane $\omega$ through $A$ meets $a_{i}$ in $A_{i}$ and contains a unique point $Q$ such that the 5 lines $Q A_{i}$ are projective with $g$. As $\omega$ describes the bundle $A_{0}, Q$ describes a cubic surface $F_{3}: A_{0} 4 a_{i}$. If $A_{0}$ describes a line $u, F_{3}$ describes a pencil. Any point $y$ of space determines a point $A_{0}$ on $u$, and the line $A_{0} y$ meets the associated $F_{3}$ in $y^{\prime}$. The involution $\left(y, y^{\prime}\right)$ is of order 11 . Its complete table of characteristics is determined. In the second transformation any plane through $u$ determines a conic having a given cross ratio at $A_{1} \cdots A_{4}$. The transversal through $y$ meeting $u$ and a sixth line $u^{\prime}$ meets the conic in $y^{\prime}$. This involution of order 9 has only straight lines for fundamental lines, both of the first and of the second kind. (Received October 30, 1931.)

405. Professor G. T. Whyburn: On the decomposability of closed sets into a countable number of simple sets of various types.

For any closed set $C$ and any class $K$ of closed sets, denote by $K(C)$ the set of all points $x$ of $C$ such that no neighborhood of $x$ is contained in any $K$-set in 
$C$. Set $C_{1}^{k}=K(C), C_{2}^{k}=K\left(C_{1}^{k}\right), \cdots, C_{\alpha}^{k}=K\left(C_{\alpha-1}^{k}\right)$ or $\prod_{\beta<\alpha} C_{\beta}^{k}$, according as $\alpha$ does or does not have an immediate predecessor, $\cdots$. We prove the following general theorem: A self-compact metric set $C$ is the sum of a countable number of $K$-sets if and only if $C_{\alpha}^{k}=0$ for some ordinal number $\alpha$ of the first or second class. An alternate form of this condition is that every subset $N$ of $C$ contain at least one point $x$ for some neighborhood $R$ of which the set $N \cdot R$ is a subset of some $K$-set in $C$. By taking $K$ to be the class of all arcs, we have a solution of the well known problem of finding necessary and sufficient conditions that a given continuum be the sum of a countable number of arcs. Other choices of $K$ are discussed, as are also the cases where $C_{n}^{k}$ vanishes $(n=1,2, \cdots)$ for general and specific $K$ 's. (Received October 24, 1931.) 